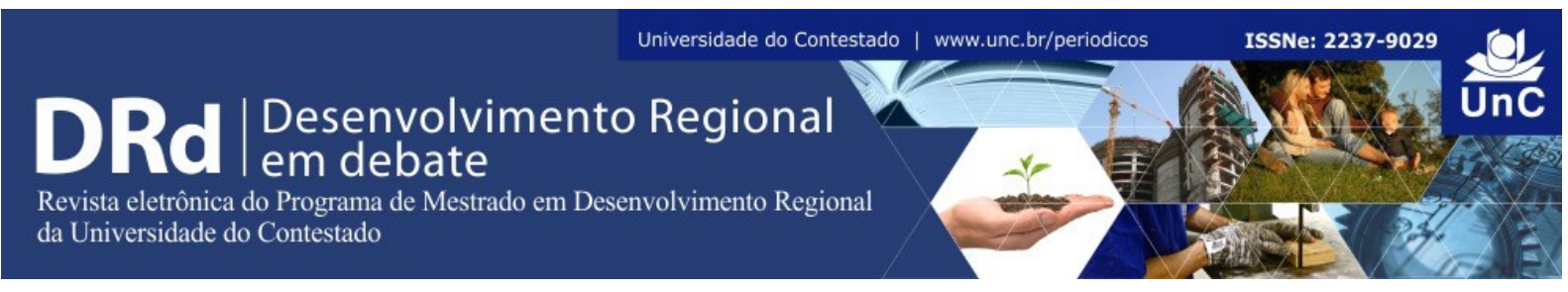

\title{
A INFLUÊNCIA DA CULTURA NA ADOÇÃO DE INOVAÇÕES TECNOLÓGICAS POR ESTUDANTES DO ENSINO MÉDIO DAS ESCOLAS PRIVADAS DE FLORIANÓPOLIS: UM ESTUDO DE CASO
}

José Lindolfo Deschamps Junior ${ }^{1}$

Alexssandro Pereira de Couto ${ }^{2}$

Denise Del Prá Netto Machado ${ }^{3}$

Nei Antonio Nunes ${ }^{4}$

\begin{abstract}
RESUMO
O presente estudo visa identificar a influência da cultura na adoção de inovações tecnológicas nos estudantes da rede privada de educação em Florianópolis, a capital do Estado de Santa Catarina. Foi realizada uma pesquisa com a aplicação de 250 questionários em alunos do $1^{\circ}$ ao $3^{\circ}$ ano do ensino médio de uma rede de escolas privadas na cidade de Florianópolis. Este estudo traz uma abordagem quantitativa com a análise de frequência das respostas e o uso da técnica de Análise de Variância ou ANOVA. Como resultados percebeu-se a tendência dos estudantes ao individualismo e a baixa distância do poder. $\mathrm{Na}$ dimensão de adoção de inovação tecnológica, os índices demonstraram uma alta tendência dos estudantes à adoção de inovações.
\end{abstract}

Palavras-chaves: Inovação Tecnológica. Dimensões da Cultura. Instituição de Ensino.

\section{THE INFLUENCE OF CULTURE IN THE ADOPTION OF TECHNOLOGICAL INNOVATIONS BY STUDENTS OF THE HIGH SCHOOL OF FLORIANÓPOLIS PRIVATE SCHOOLS: A CASE STUDY}

\begin{abstract}
The present study aims to identify the influence of culture in the adoption of technological innovations in students of the private education network in Florianópolis. A survey was carried out with the application of 250 questionnaires in students from the 1st to 3rd year of high school in a network of private schools in the city of Florianópolis. This study provides a quantitative approach with the frequency analysis of the responses and the use of the Analysis of Variance or ANOVA technique. As a result, students' tendency to individualism and the low power distance were perceived. In the dimension of adoption of technological innovation, the indices showed a high tendency of the students to adopt innovations.
\end{abstract}

Key Words: Technologic Innovation. Dimensions of Culture. Educational Institution.

\footnotetext{
${ }^{1}$ Mestrando em Administração na Universidade do Sul de Santa Catarina (UNISUL). Universidade do Sul de Santa Catarina (UNISUL). Brasil. E-mail: juniordeschamps@gmail.com.

${ }^{2}$ Mestrando em Administração na Universidade do Sul de Santa Catarina (UNISUL). Universidade do Sul de Santa Catarina (UNISUL). Brasil. E-mail: alexssandropcouto@gmail.com

${ }^{3}$ Doutora em Administração de Empresas pela Fundação Getúlio Vargas - SP - EAESP/FGV, com atuação na Universidade do Sul de Santa Catarina (UNISUL). Brasil. E-mail: profadenisedelpra@gmail.com

${ }^{4}$ Mestre em Educação (UFSC) e Doutor em Sociologia Política (UFSC). Atua na Universidade do Sul de Santa Catarina (UNISUL). Brasil. E-mail: nei.nunes@unisul.br
}

DRd - Desenvolvimento Regional em debate (ISSNe 2237-9029)

v. 9, p. 42-58, 2019. 


\section{INTRODUÇÃO}

A inovação é um processo, produto ou serviço que pode apresentar características inéditas ou já conhecidas, e que venham a promover melhorias significativas em seu desempenho. Devido a sua amplitude, a inovação se torna uma temática de estudo importante para várias áreas de conhecimento, como tecnologia, design, engenharias etc. Nas últimas décadas este assunto vem ganhando destaque nos estudos organizacionais, em especial por estar vinculado à competitividade e ao desenvolvimento

Segundo Nicolaci-Da Costa (2005), as inovações tecnológicas penetraram em nossas vidas com os computadores e a internet, celulares e outros produtos e aparelhos usados adultos e jovens. Em estudo feito com jovens foram identificadas alterações no aumento de sua autonomia, liberdade e privacidade. Também se observou o incremento da intimidade em vários de seus relacionamentos, o aumento de segurança e o sentimento de nunca estarem sozinhos.

As inovações tecnológicas veem sendo utilizadas em escala crescente e interferindo no cotidiano de pessoas de todos continentes. Por mais que no Brasil tenhamos realidades distintas em aquisição de inovações tecnológicas, entende-se que existe uma considerável importância no reconhecimento da adoção de inovações por estudantes, principalmente no que tange às diferenças sociais.

A instituição de ensino e o grupo de amigos, para o adolescente, funcionam como um espaço intermediário entre a família e a sociedade. Por mais que a família exerça uma grande influência no comportamento e na decisão dos jovens o grupo social que ele faz parte também impacta fortemente em suas preferências. Considera-se neste contexto, que a escola forme o agrupamento social que, muitas vezes, irá influenciar na visão de mundo do estudante.

A visão de mundo pode ser caracterizada como a forma que determinado grupo social define aquilo que é aceito e que o caracteriza. Esta visão delimita seus valores, pressupostos e, por meio de rituais, as condutas de como cada elemento deve se comportar. Algumas regras, escritas ou não, também fazem parte do direcionamento que irá determinar quem pertence ou não ao grupo. A esta visão de mundo chamamos de cultura.

Este artigo objetiva identificar a influência da cultura na adoção de inovações tecnológicas por estudantes das escolas privadas da capital do Estado de Santa Catarina. Para a identificação da influência da cultura na adoção de inovações, serão utilizados os conceitos de distância do poder e individualismo versus coletivismo de Hofstede (1991).

O trabalho está estruturado da seguinte forma: nesta primeira seção foram introduzidos o tema e o objetivo de pesquisa e na próxima seção, serão apresentados os conceitos de inovação, inovação tecnológica e cultura organizacional. Posteriormente, são apresentados os procedimentos metodológicos utilizados e a caracterização da população pesquisada e, por fim, os resultados, as conclusões e sugestões para estudos futuros.

DRd - Desenvolvimento Regional em debate (ISSNe 2237-9029) 


\section{REFERENCIAL TEÓRICO}

\subsection{INOVAÇÃO}

Vasconcellos (2003) caracteriza a inovação como fundamental para o desenvolvimento e competitividade das organizações a partir de 1990 identificando que a inovação teve importante papel para o sucesso das organizações. Para se tornarem mais competitivas, precisam não apenas apresentar um maior portfólio de produtos. Elas precisam criar algo novo que venha ao encontro das necessidades dos consumidores. Para o autor, a organização inovadora é aquela que realiza inovações com autonomia, intencionalidade e proatividade. A inovação é parte fundamental da existência da organização, desenvolvendo e implementando recursos tangíveis e intangíveis de forma perene ao longo do seu ciclo de vida.

O Manual de Oslo (OCDE, 2005) menciona que Joseph Schumpeter influenciou consideravelmente as teorias da inovação. $\mathrm{O}$ desenvolvimento econômico é conduzido pela inovação por meio de um processo dinâmico no qual as novas tecnologias substituem as antigas, denominada "destruição criadora". Segundo Schumpeter (2003), inovações "radicais", aquelas vindas de fora da organização, engendram rupturas mais intensas, enquanto inovações "incrementais", consideradas por ele como internas, dão continuidade ao processo de mudança.

Schumpeter (2003) propôs uma lista de cinco tipos de inovação engendradas pelas organizações: i) introdução de novos produtos; ii) introdução de novos métodos de produção; iii) abertura de novos mercados; iv) desenvolvimento de novas fontes provedoras de matériasprimas e outros insumos e; v) criação de novas estruturas de mercado em uma indústria. Para o autor, um material, uma ideia ou uma prática, concebidos ou percebidos como novos, possuindo uma aplicação relevante, são considerados como inovação. Essa concepção insere as mudanças ou melhorias, indicando inovações incrementais ou com baixa escala tecnológica (ROTHWELL; GARDINER, 1985).

Um dos motivos que leva uma organização a inovar é a possibilidade de melhoria no seu desempenho, que pode se dar pelo aumento da demanda ou a redução dos custos. A criação de um novo produto ou processo pode trazer vantagem competitiva, possibilitando aumento da produtividade e uma maior margem sobre os custos, o que ajuda a elevar os lucros da organização (SCHUMPETER, 2003).

Mesmo sendo este um tema estudado há décadas por autores como Schumpeter (2003), Rogers e Shoemaker (1971), Zaltman, Duncan e Holbeck (1973), Downs e Mohr (1976), Dosi (1982, 1988), Van de Ven e Angle (1989), Damanpour (1991), Hatch (1997), Tidd, Bessant e Pavitt (2005), Gundling (1999), Afuah (2003), Hamel (2006), Ismail e Abdmajid (2007), entre outros, a literatura sobre o tema abrange diversas áreas além de ser uma temática complexa e multidimensional. O consenso sobre inovação é de ser fundamental para a competitividade. Segundo Varandas et. al. (2014) a inovação se tornou fundamental para a geração de valor e sustentabilidade das organizações contemporâneas, tanto local quanto em nível internacional. Ela tem o poder de alavancar receitas e elevar o lucro da organização, melhorar as relações com os stakeholders, bem como a capacidade de contribuir

DRd - Desenvolvimento Regional em debate (ISSNe 2237-9029)

v. 9, p. 42-58, 2019. 
para a motivação dos funcionários, não sendo possível crescer em um ambiente competitivo apenas pela redução de custos ou melhoria em processos.

\subsubsection{Inovação Tecnológica}

O precursor da inovação tecnológica é sem dúvida Schumpeter (2003). O autor reserva seu conceito de inovação ao comportamento empreendedor, o qual possui considerável impacto ao desenvolvimento econômico e às transformações tecnológicas. A organização, por meio de seu investidor, se embrenha no desenvolvimento de inovações técnicas que, em consequência, irão facilitar o desenvolvimento econômico das regiões onde estas estão inseridas ou as tecnologias consumidas. Neste contexto de empreender e da mudança e geração da técnica, se dá a inovação tecnológica.

Segundo Casagrande (2015), a inovação tecnológica gera mudanças e pode representar desafios, bem como gerar insegurança e instabilidade para administradores conservadores. Ser criativo é fundamental para a introdução de novos produtos ou serviços.

Para Vargas, Schiavini e Schreiner (2012), o desenvolvimento da tecnologia é um fator determinante da competitividade. Através da constante inovação nos processos, a organização pode manter seu posicionamento no mercado e até superar seus concorrentes. A inovação pode contribuir para proteger as organizações contra cópia dos concorrentes.

Para Breschi e Malerba (1997, p. 132) existem quatro características definidoras de um regime tecnológico que definirá a trajetória de uma inovação tecnológica: "Regime tecnológico é definido pelo nível e tipo de condições de oportunidade e apropriabilidade, pela cumulatividade do conhecimento tecnológico, pela natureza do conhecimento e pelos meios de transmissão e comunicação de conhecimento".

O nível de oportunidade é caracterizado por dois aspectos básicos: a oportunidade propriamente dita e a capilaridade que a tecnologia possui. A oportunidade se resume a quantidade de oportunidades que são geradas no ambiente externo da organização. A Capilaridade diz respeito a quanto há de difusão do conhecimento concernente àquela inovação. Ou seja, a amplitude que aquela inovação alcança em sua trajetória. Apropriabilidade se refere a capacidade da organização de defender e proteger suas inovações do mercado competidor. Neste sentido, são utilizadas ferramentas como o patenteamento, os segredos industriais, o incentivo a ambientes internos à inovação como treinamentos etc. Cumulatividade é a capacidade de gestão e a trajetória do conhecimento tecnológico. Este conhecimento irá transitar pelos níveis individual, tecnológico, organizacional e da indústria referência. A Base de conhecimento incorpora a complexidade da tecnologia e quanto do conhecimento é tácito. Assim, há uma maior complexidade dependente das inter-relações na geração do conhecimento e grau de codificação e acesso deste conhecimento (BRESCHI; MALERBA, 1997).

No Brasil, a Lei de Inovação Tecnológica (Lei Federal n. ${ }^{\circ}$ 10.973/2004), que “dispõe sobre incentivos à inovação e à pesquisa científica e tecnológica no ambiente produtivo e dá outras providências", foi a precursora no incentivo legal para o desenvolvimento tecnológico.

DRd - Desenvolvimento Regional em debate (ISSNe 2237-9029) 
Conforme Matias-Pereira e Kruglianskas (2005), a Lei de Inovação Tecnológica se organiza em três eixos que darão sustentabilidade ao Sistema Nacional de Inovação (SNI), composto por Empresas, Governo e Universidades. O primeiro eixo é a constituição de um ambiente que propicie parcerias estratégicas entre os componentes do SNI. O segundo é a criação de estímulos para que as Instituições de ciência e tecnologia possam participar de processos de inovação. O terceiro é o incentivo à inovação empresarial, com estímulo por meio de financiamentos e bolsas de pesquisa na empresa. Forma-se, portanto, condições de se inovar tecnologicamente no Brasil.

\subsection{CULTURA ORGANIZACIONAL}

Este trabalho utilizará o conceito de cultura organizacional para o comportamento coletivo de grupos sociais inseridos em um determinado contexto. No caso específico deste trabalho, para estudantes de escolas particulares da capital do Estado de Santa Catarina.

Esta opção se baseia no trabalho de Becker e Geer (1960), que utilizaram o conceito de "cultura organizacional" argumentando que existe uma cultura latente, oriunda de grupos sociais dos quais os membros participam. Estes grupos são externos à organização formal e afetam diretamente o comportamento do indivíduo. Em qualquer situação de grupo, aquilo que o indivíduo é e o modo como é visto afeta a forma como ele se comporta.

Para Pettigrew (1979, p. 581), a cultura é o conjunto de significados aceitos por um grupo em um determinado momento. Desta forma a cultura se refere a algo mais abrangente pois estabelece um cenário que define o que é certo e errado, dirige a moral, leis e costumes para indivíduos interagirem socialmente de forma adequada.

Conforme Machado e Carvalho (2006), os valores, crenças, modo de agir e perceber os acontecimentos podem definir o termo cultura organizacional. Cada organização cria sua própria cultura ou clima, seus próprios tabus, usos e costumes, podendo ter origem tanto nas normas e valores formais quanto informais.

Segundo Pires e Macêdo (2006), torna-se fundamental a compreensão da cultura organizacional para a construção da estrutura das organizações. A cultura organizacional pode ser percebida como um conjunto de características que as distingue em relação às demais. Essa cultura, assume o papel de legitimadora do sistema de valores, expressando-se através de rituais, mitos, hábitos e crenças comuns aos membros de uma organização, produzindo normas de comportamento genericamente aceitas por todos.

$\mathrm{Na}$ visão de Hofstede (1991), cultura organizacional é definida como a maneira em que membros de uma organização se relacionam entre si, o seu trabalho e o mundo exterior, em comparação com outras organizações, desempenhando um papel significativo na formação de nossos modos de sentir, pensar e agir. No mundo, as culturas diferem das nações e regiões. Ele desenvolveu a teoria da dimensão cultural para explicar as diferenças culturais nacionais. Através destas dimensões, é possível entender como as culturas nacionais funcionam. Para isso, realizou uma ampla pesquisa em 40 países no período de quinze anos, a qual resultou no

DRd - Desenvolvimento Regional em debate (ISSNe 2237-9029) 
reconhecimento de quatro dimensões que explicam cerca de $50 \%$ das diferenças entre os padrões de valores. No Quadro 1 apresentamos essas dimensões e suas características.

Quadro 1 - As dimensões de cultura de Hofstede

\begin{tabular}{|l|l|}
\hline \multicolumn{1}{|c|}{ Dimensões } & \multicolumn{1}{|c|}{ Características } \\
\hline Distância de poder & $\begin{array}{l}\text { Extensão na qual a sociedade aceita o fato de que o poder é distribuído } \\
\text { desigualmente nas organizações e na própria sociedade, onde existem } \\
\text { privilégios e a autoridade pessoal absoluta é aceita. }\end{array}$ \\
\hline Individualismo x Coletivismo & $\begin{array}{l}\text { A maneira como o indivíduo está ligado à estrutura social; se esse laço é mais } \\
\text { rígido ou mais flexível, no sentido de oferecer oportunidades para a exibição de } \\
\text { comportamentos individualistas. Basicamente, a ideia do nós em contraposição } \\
\text { ao eu. Individualismo significa uma sociedade em que os laços entre os } \\
\text { indivíduos estão solto. Coletivismo significa uma sociedade em que as pessoas } \\
\text { são interligadas desde o nascimento, coesas em grupos e ao longo da vida, } \\
\text { continuam a protegê-los em troca de lealdade inquestionável. }\end{array}$ \\
\hline Evitar incertezas & $\begin{array}{l}\text { O medo da mudança é o grau em que os membros de uma cultura se sentem } \\
\text { ameaçados por situações ambíguas ou desconhecidas. As sociedades } \\
\text { apresentam diferentes coeficientes de aversão ao risco. }\end{array}$ \\
\hline Masculinidade / Feminilidade & $\begin{array}{l}\text { Extensão na qual a sociedade privilegia a realização, heroísmo, determinação } \\
\text { pessoal e sucesso material, em oposição à preferência por relacionamentos, } \\
\text { modéstia, cuidado com os outros, qualidade de vida etc. }\end{array}$ \\
\hline $\begin{array}{l}\text { Orientação de Curto / Longo } \\
\text { Prazo }\end{array}$ & $\begin{array}{l}\text { Orientação de curto prazo está para a promoção de virtudes relacionadas com o } \\
\text { passado e presente, respeito a tradição, a preservação da “face”, e cumprindo } \\
\text { obrigações sociais. } \\
\text { Orientação de longo prazo significa a promoção de virtudes orientadas para } \\
\text { recompensas futuras, em especial perseverança e frugalidade. }\end{array}$ \\
\hline
\end{tabular}

Fonte: Elaborado pelos autores, 2018.

$\mathrm{Na}$ visão de Hofstede (1991), os graus de igualdade, ansiedade, poder, incerteza, feminilidade explicam a estrutura das organizações. As regras que direcionam uma nação, serão as guias que também conduzirão as organizações pertencentes a ela.

\section{METODOLOGIA}

Considerando que o objetivo deste estudo é identificar a influência da cultura na adoção de inovações tecnológicas por estudantes do ensino médio da cidade de Florianópolis, capital do Estado de Santa Catarina, foi escolhida para este estudo uma pesquisa descritiva com corte transversal, ou seja, um recorte temporal.

Trata-se de uma pesquisa quantitativa, com a finalidade de analisar as correlações entre cultura dos estudantes e a adoção de inovações tecnológicas. Portanto, seguindo os preceitos de Hair Jr. et al. (2005), serão isoladas as variáveis-chave. São elas a Cultura e a adoção de inovações tecnológicas. A Cultura será analisada por meio das dimensões de Hofstede (1991) distância do poder e coletivismo versus individualismo. A adoção de inovações tecnológicas terá como modelo a proposta de Parasuramann (2000), já utilizado no trabalho de Gonçalves et al. (2016).

A população deste estudo é composta por 25.600 estudantes matriculados nas escolas privadas da cidade de Florianópolis, segundo o Sindicato Nacional das Escolas Particulares de 
Santa Catarina. Como amostra, foi aplicado o questionário com 250 estudantes do ensino médio, do primeiro, segundo e terceiro ano, pertencentes a uma rede de escolas privadas da capital de Santa Catarina no período matutino. A amostra foi não probabilística por acessibilidade (HAIR Jr. et al., 2005).

O instrumento utilizado para a coleta dos dados foi questionário utilizado e validado por Gonçalves et al. (2016), com 19 questões, com opções de resposta em escala tipo Likert de 7 pontos que estratificam os elementos da cultura que influenciam na adoção de novas tecnologias pelos jovens de Florianópolis. O modelo completo é formado por três partes. A primeira parte se refere a dimensão distância do poder com as questões Q1, Q2 e Q3. A segunda parte objetiva identificar o grau de coletivismo versus individualismo com as questões Q4, Q5, Q6, Q7, Q8 e Q9. A adoção de inovações foi mensurada pelas questões Q10 até Q19.

Os dados obtidos através dos questionários, foram tabulados em planilha do software Microsoft Excel, sendo as perguntas agrupadas nas dimensões às quais pertencem. Após tabulados, foi feita uma análise da frequência das respostas da escala tipo Likert de cada pergunta para montar o perfil e a característica dos entrevistados, através de uma análise descritiva.

Para confiabilidade das dimensões, na segunda etapa, foi utilizado o alfa de Cronbach. Conforme recomendação de Hair Jr. et al. (2005) somente serão considerados válidos os resultados com valores superiores a 0,60 . Por último, para averiguar a influência da cultura sobre a adoção de inovações, foi utilizada a técnica de Analise de Variância ou ANOVA, que é uma metodologia estatística com abordagem confirmatória.

\section{ANÁLISE DOS RESULTADOS}

Conforme a Tabela 1, são apresentados o gênero e idade dos respondentes.

Tabela 1 - Gênero e idade dos respondentes

\begin{tabular}{ccc}
\hline & GÊNERO & FREQUENCIA \\
\hline Masculino & & 139 \\
\hline Feminino & IDADE & 111 \\
\hline 16 & FREQUÊNCIA \\
\hline 17 & $8 \%$ \\
\hline 18 & $33,62 \%$ \\
\hline 19 & $14,39 \%$ \\
\hline 20 & $13,20 \%$ \\
\hline TOU MAIS & $13,20 \%$ \\
\hline TOTAL & $17,59 \%$ \\
\hline
\end{tabular}

Fonte: Dados da Pesquisa (2018)

Conforme a Tabela 1, na rede privada a maioria dos respondentes são do sexo masculino. A faixa etária dos respondentes varia entre 16 e 25 anos. Salienta-se que $58,38 \%$

DRd - Desenvolvimento Regional em debate (ISSNe 2237-9029) 
dos estudantes possuem mais que dezoito anos. Segundo a direção da escola este fato ocorre pela busca do terceiro ano do segundo grau como um reforço pré-vestibular.

$\mathrm{Na}$ Tabela 2 que busca identificar com quais familiares os estudantes residem.

Tabela 2 - Residência do estudante

\begin{tabular}{lcc}
\hline & RESIDE COM & FREQUÊNCIA \\
\hline Mãe e Pai & 156 \\
\hline Mãe & 56 \\
\hline Pai & 8 \\
\hline Outros & 30 \\
\hline TOTAL & $\mathbf{2 5 0}$ \\
\hline
\end{tabular}

Fonte: Dados da Pesquisa (2018)

Se observou, dentre os 250 pesquisados da rede privada de ensino, que 156 jovens moram com a mãe e o pai, 56 estudantes moram com a mãe, 8 estudantes com os pais e 30 estudantes moram com outras pessoas.

Na Tabela 3 são apresentados a propriedade de um ou mais imóveis.

Tabela 3 - Propriedade do Imóvel

\begin{tabular}{lcc}
\hline & IMÓVEL & FREQUÊNCIA \\
\hline Próprio & 213 \\
\hline Alugado & 37 \\
\hline TOTAL & 250 \\
\hline Na Praia & $119-47,6 \%$ do total \\
\hline
\end{tabular}

Fonte: Dados Pesquisas (2018)

Observa-se na Tabela 3 que 213 estudantes possuem residência própria e 37 residem em imóveis alugados. Além disso, 119 estudantes apontaram que a família possui imóvel na praia.

Na Tabela 4 é apresentado o grau de instrução dos pais dos estudantes.

Tabela 4 - Instrução Pai e Mãe

\begin{tabular}{|c|c|c|c|}
\hline INSTRUÇÃO MÃE & FREQUÊNCIA & INSTRUÇÃO PAI & FREQUÊNCIA \\
\hline FUNDAMENTAL & $4 \%$ & FUNDAMENTAL & $3,20 \%$ \\
\hline MÉDIO & $15,2 \%$ & MÉDIO & $14,40 \%$ \\
\hline SUPERIOR INC. & $6,4 \%$ & SUPERIOR INC. & $10 \%$ \\
\hline SUPERIOR COMP. & $38,4 \%$ & SUPERIOR COMP. & $31,21 \%$ \\
\hline PÓS-GRAD. & $36 \%$ & PÓS-GRAD. & $41,19 \%$ \\
\hline TOTAL & $100 \%$ & & $100 \%$ \\
\hline
\end{tabular}

Fonte: Dados da Pesquisa (2018)

Observa-se que 19,2\% das mães e 17,6\% dos pais possuem ensino médio. Em relação ao Ensino Superior as mães apontam uma maior formação, totalizando $74,4 \%$ das respondentes enquanto totalizam $72,4 \%$ os pais com ensino superior. 
Após uma análise geral dos dados demográficos dos estudantes, serão apresentados os dados das variáveis desta pesquisa: dimensões da cultura e adoção de inovações. Na tabela 5 se apresenta a Dimensão Distância do Poder.

Tabela 5 - Resultados (D1) - Dimensão Distância do Poder

\begin{tabular}{|c|c|c|c|c|c|c|c|c|}
\hline $\begin{array}{l}\text { 1- Discordo totalmente } 7 \text { - concordo } \\
\text { totalmente }\end{array}$ & 1 & 2 & 3 & 4 & 5 & 6 & 7 & Média \\
\hline $\begin{array}{l}\text { 1. Em sua casa, seus pais devem tomar } \\
\text { a maioria das decisões sem consultar } \\
\text { você. }\end{array}$ & $43,2 \%$ & $24,4 \%$ & $11,6 \%$ & $11,2 \%$ & $5,2 \%$ & $2,4 \%$ & $2 \%$ & 2,16 \\
\hline $\begin{array}{l}\text { 2. Eu não devo discordar das decisões } \\
\text { dos meus pais. }\end{array}$ & $38,8 \%$ & $18,4 \%$ & $13,6 \%$ & $15,2 \%$ & $6,8 \%$ & $3,6 \%$ & $3,59 \%$ & 2,46 \\
\hline $\begin{array}{l}\text { 3. Nossa família deve evitar se } \\
\text { envolver com pessoas de outra classe } \\
\text { social. }\end{array}$ & $86,8 \%$ & $8 \%$ & $2,8 \%$ & $1,2 \%$ & $0,4 \%$ & - & $0,8 \%$ & 1,23 \\
\hline
\end{tabular}

Fonte: Dados da Pesquisa (2018)

A distância do poder pode ser definida como a forma que a sociedade aceita o fato de que o poder é distribuído desigualmente nas organizações e na própria sociedade, na qual existem privilégios e a autoridade pessoal absoluta é aceita (HOFSTEDE, 2011). O resultado das questões desta dimensão demonstrou uma baixa distância do poder, havendo discordância das questões respondidas. Na escala tipo Likert de 1 a 7 , sendo 1 discordo totalmente e 7 concordo totalmente, a média desta dimensão foi de 1.95 pontos na escala. Pelos percentuais, $81 \%$ dos respondentes discordam da alta distância do poder dentro de casa. Na Q3, 97,6\% dos estudantes discordam no quesito que a família deve evitar se envolver com pessoas de outra classe social. Tais resultados demonstram a posição dos jovens de pequena distância do poder e que as desigualdades entre as pessoas devem ser minimizadas.

Tabela 6 - Resultados (D2) - Dimensão Individualismo vs Coletivismo

\begin{tabular}{|c|c|c|c|c|c|c|c|c|}
\hline $\begin{array}{l}\text { 1-discordo totalmente } 7 \text { - concordo } \\
\text { totalmente }\end{array}$ & 1 & 2 & 3 & 4 & 5 & 6 & 7 & Média \\
\hline $\begin{array}{l}\text { 4. Eu devo esquecer (abrir mão) do } \\
\text { que quero se isto prejudicar meu } \\
\text { grupo de estudos (trabalhos de escola). }\end{array}$ & $19,6 \%$ & $12,8 \%$ & $16 \%$ & $24,4 \%$ & $10,8 \%$ & $9,2 \%$ & $7,2 \%$ & 3,36 \\
\hline $\begin{array}{l}\text { 5. Eu devo deixar de lado o que quero } \\
\text { para seguir o que meu grupo de } \\
\text { estudos quer (trabalhos de escola). }\end{array}$ & $59,19 \%$ & $16 \%$ & $8,4 \%$ & $8,4 \%$ & $3,2 \%$ & $2,8 \%$ & $2,1 \%$ & 1,96 \\
\hline $\begin{array}{l}\text { 6. Eu devo ficar com meu grupo de } \\
\text { estudos (trabalhos de escola) mesmo } \\
\text { quando ele estiver em dificuldades. }\end{array}$ & $10 \%$ & $4,39 \%$ & $5,6 \%$ & $8,4 \%$ & $10 \%$ & $22,8 \%$ & $38,81 \%$ & 5,05 \\
\hline $\begin{array}{l}\text { 7. O bem-estar do meu grupo de } \\
\text { estudos (trabalhos de escola) é mais } \\
\text { importante do que as recompensas } \\
\text { individuais que eu possa receber. }\end{array}$ & $18 \%$ & $8,4 \%$ & $13,6 \%$ & $32,4 \%$ & $6 \%$ & $11,2 \%$ & $10,4 \%$ & 3,5 \\
\hline $\begin{array}{l}\text { 8. O sucesso do meu grupo de estudos } \\
\text { (trabalhos de escola) é mais } \\
\text { importante que o meu sucesso } \\
\text { individual. }\end{array}$ & $37,2 \%$ & $18,8 \%$ & $14 \%$ & $14,79 \%$ & $6,4 \%$ & $5,2 \%$ & $4,61 \%$ & 2,56 \\
\hline $\begin{array}{l}\text { 9. Só devo perseguir meus objetivos } \\
\text { depois de considerar o que é melhor } \\
\text { para o meu grupo de estudos } \\
\text { (trabalhos de escola) }\end{array}$ & $34,8 \%$ & $22 \%$ & $14 \%$ & $15,6 \%$ & $2,8 \%$ & $5,2 \%$ & $5,6 \%$ & 2,48 \\
\hline
\end{tabular}

DRd - Desenvolvimento Regional em debate (ISSNe 2237-9029) 
A dimensão individualismo vs coletivismo refere-se à preferência de um grupo por ações individuais ao invés de ações em grupo e é identificada nas respostas 4 a 9 do questionário. As questões foram elaboradas com afirmações que reforçam o coletivismo e os estudantes responderam se concordavam totalmente ou discordam totalmente, assim quanto maior o resultado do indicador, maior o índice de coletivismo.

A dimensão apresentou uma média de 2,7 pontos na escala tipo Likert, demonstrando a tendência dos estudantes a uma cultura mais individualista. A tendência dos estudantes a esta cultura fica mais forte analisando-se a questão 5, na qual 59,9\% discordam que devem deixar de lado o que querem para seguir o que seu grupo de estudos quer. A questão 8 também demonstra uma cultura mais individualista com $70 \%$ dos estudantes discordando da afirmativa que o sucesso do grupo de estudos é mais importante que o sucesso individual.

A terceira parte do questionário, apresentada na Tabela 7 corresponde a 10 questões que visam identificar a adoção tecnológica pelos estudantes. Este questionário foi adaptado de Parasuramann (2000), que objetivou mensurar a disposição das pessoas em adotar e usar novas tecnologias. O questionário utilizado neste estudo como modelo de coleta de dados foi utilizado por Gonçalves et al. (2016).

Tabela 7 - Resultados (D3) - Nível de Adoção Tecnológica

\begin{tabular}{|c|c|c|c|c|c|c|c|c|}
\hline $\begin{array}{l}\text { 1- Discordo totalmente } 7 \text { - concordo } \\
\text { totalmente }\end{array}$ & 1 & 2 & 3 & 4 & 5 & 6 & 7 & Média \\
\hline $\begin{array}{l}\text { 10. Meus colegas me procuram para } \\
\text { pedir conselhos sobre novidades em: } \\
\text { telefones celulares, tablets, aplicativos, } \\
\text { computadores, jogos eletrônicos e } \\
\text { outras tecnologias }\end{array}$ & $23,61 \%$ & $10,4 \%$ & $14,39 \%$ & $16 \%$ & $13,2 \%$ & $5,6 \%$ & $16,8 \%$ & 3,67 \\
\hline $\begin{array}{l}\text { 11. Parece que meus amigos estão } \\
\text { aprendendo mais novidades em: } \\
\text { telefones celulares, tablets, aplicativos, } \\
\text { computadores, jogos eletrônicos e } \\
\text { outras tecnologias do que eu. * }\end{array}$ & $6,8 \%$ & $11,6 \%$ & $10,4 \%$ & $20 \%$ & $10,4 \%$ & $15,2 \%$ & $25,6 \%$ & 4,63 \\
\hline $\begin{array}{l}\text { 12. Em geral, eu estou entre os } \\
\text { primeiros de meus amigos que } \\
\text { conhecem as novidades em: telefone } \\
\text { celulares, tablets, aplicativos, } \\
\text { computadores, jogos eletrônicos e } \\
\text { outras tecnologias }\end{array}$ & $20,4 \%$ & $14 \%$ & $15,2 \%$ & $14 \%$ & $19,6 \%$ & $6,8 \%$ & $10 \%$ & 3,57 \\
\hline $\begin{array}{l}\text { 13. Normalmente, consigo descobrir } \\
\text { novidades em: telefones celulares, } \\
\text { tablets, aplicativos, computadores, } \\
\text { jogos eletrônicos e outras tecnologias, } \\
\text { sem a ajuda de outras pessoas. }\end{array}$ & $4,8 \%$ & $3,59 \%$ & $7,6 \%$ & $11,2 \%$ & $12 \%$ & $29,2 \%$ & $31,59 \%$ & 5,16 \\
\hline $\begin{array}{l}\text { 14. Consigo acompanhar as novidades } \\
\text { em: telefones celulares, tablets, } \\
\text { aplicativos, computadores, jogos } \\
\text { eletrônicos e outras tecnologias que } \\
\text { mais me interessam. }\end{array}$ & $4 \%$ & $2,4 \%$ & $5,2 \%$ & $8,79 \%$ & $11,2 \%$ & $27,6 \%$ & $40,52 \%$ & 5,55 \\
\hline $\begin{array}{l}\text { 15. Me divirto com o desafio de } \\
\text { descobrir novidades em: telefones } \\
\text { celulares, tablets, aplicativos, } \\
\text { computadores, jogos eletrônicos e }\end{array}$ & $6,4 \%$ & $8,4 \%$ & $8 \%$ & $20,4 \%$ & $14,39 \%$ & $16 \%$ & $26,41 \%$ & 4,64 \\
\hline
\end{tabular}

DRd - Desenvolvimento Regional em debate (ISSNe 2237-9029)

v. 9, p. 42-58, 2019. 
A influência da cultura na adoção de inovações tecnológicas por estudantes do ensino médio das escolas privadas de Florianópolis: um estudo de caso

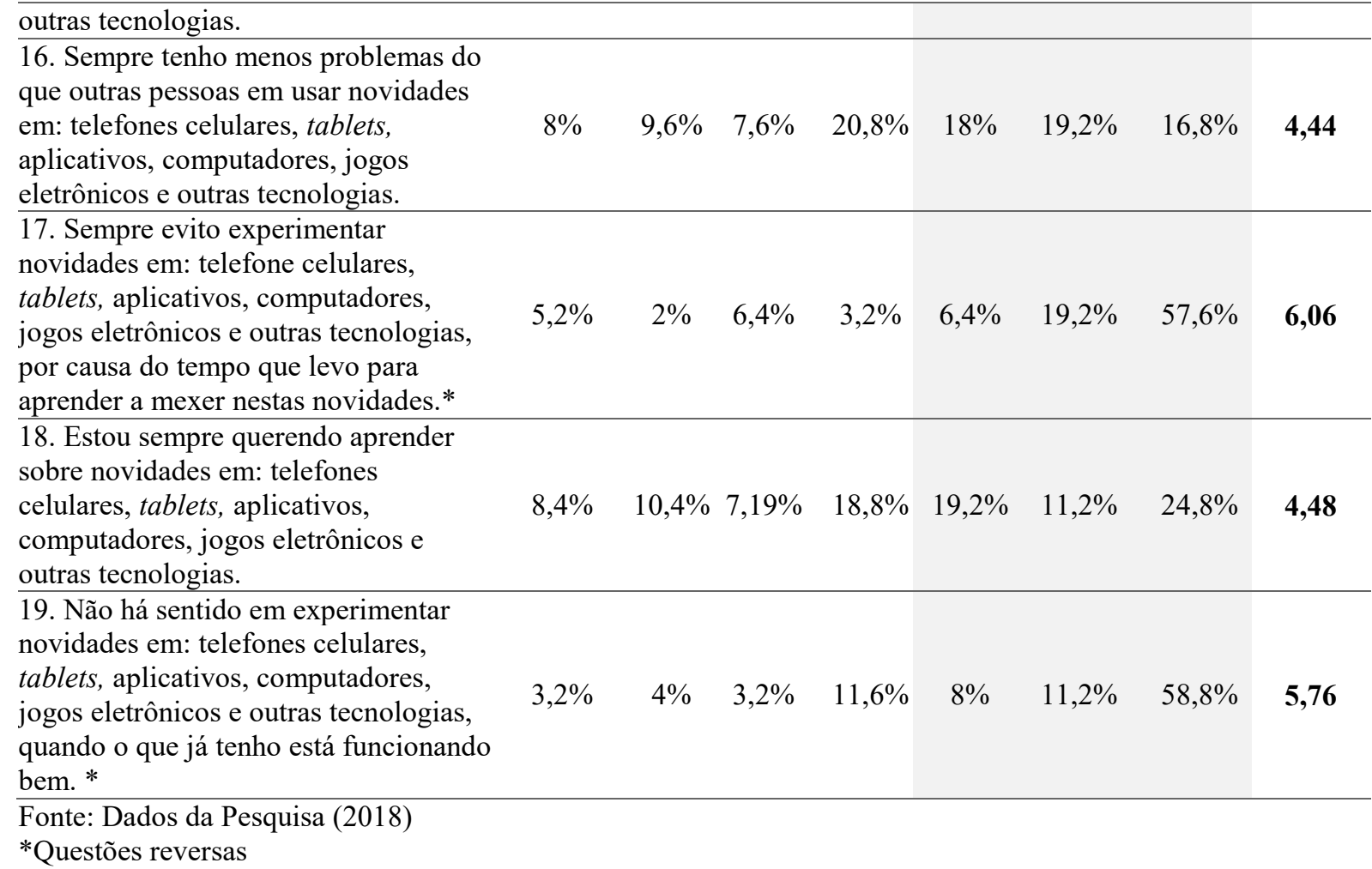

As afirmações foram elaboradas para que uma resposta com indicador mais alto identifique um estudante mais inserido digitalmente ou mais adepto a inovações tecnológicas, enquanto um indicador mais baixo identifique um estudante com menos tendência à adoção de inovações tecnológicas.

A dimensão apresentou uma média de 4,50 pontos na escala de tipo Likert, ou seja, os estudantes apresentam uma alta propensão a adoção de inovações tecnológicas. Inclusive destaca-se o resultado da afirmativa 14 que busca identificar se o estudante consegue acompanhar as novidades em telefones celulares, tablets, aplicativos, computadores, jogos eletrônicos e outras tecnologias que mais os interessam, teve uma média de 5,55 na escala tipo Likert e um total de $78 \%$ dos estudantes concordam com esta afirmativa.

Além do resultado da questão 14, as questões reversas número 17 e 19 apresentam a maior diferença de resposta no questionário, quando $80 \%$ dos jovens discordam sobre não experimentar novidades em: telefone celulares, tablets, aplicativos, computadores, jogos eletrônicos e outras tecnologias, por causa do tempo que levam para aprender a mexer nestas novidades, assim, infere-se pela análise da frequência a tendência da adoção de inovação tecnológica por estudantes da rede privada de educação.

\subsection{ANÁLISE DE CONFIABILIDADE}

Após analisar as repostas dos questionários calculando a frequência das variáveis de cultura e inovação, buscou-se verificar a confiabilidade das respostas, obtido por meio do

DRd - Desenvolvimento Regional em debate (ISSNe 2237-9029) 
alpha de Cronbach. A Tabela 8 apresenta o resultado do alfa de Cronbach das escolas particulares.

Tabela 8 - alfa de Cronbach

\begin{tabular}{lc}
\hline & Alfa de Cronbach \\
\hline Processo & Alfa de Cronbach \\
\hline Particulares &, 622 \\
\hline Total &, 622 \\
\hline Fonte: Os autores (2018) &
\end{tabular}

Segundo Hair Jr et al. (2009), o alfa de Cronbach estima o grau de convergência dos dados, assim quanto maior o grau (devendo ser maior do que 0,6 ) maior será a fidedignidade delas ou melhor será a confiabilidade das respostas da pesquisa. Segundo Malhotra (2001), caso o índice seja maior que 0,7 os dados são considerados aceitáveis e quanto mais próximo de 1, maior a sua confiabilidade. Neste estudo o valor de corte será de 0,60.

\subsection{ANÁLISE DE VARIÂNCIA}

A Tabela 9 apresenta o índice de significância (Sig.) que as variáveis independentes possuem na ANOVA. Ressalta-se que essas variáveis dependentes pertencem a cultura, cujas questões dividem-se nas dimensões: distância do poder (Q1, Q2 e Q3) e coletivismo (Q4, Q5, Q6, Q7, Q8, Q9). As afirmativas Q10, Q 11, Q12, Q13, Q 14, Q15, Q16, Q17, Q18 e Q19 conforme explicado anteriormente servem para mensurar a adoção de inovações tecnológicas e foram tratadas como variáveis independentes.

Tabela 9- Análise de Regressão

\begin{tabular}{|c|c|c|c|c|c|c|c|}
\hline \multirow{2}{*}{$\begin{array}{l}\text { Particular } \\
\text { Florianópolis }\end{array}$} & \multicolumn{2}{|c|}{$\begin{array}{c}\text { Coeficientes não } \\
\text { padronizados }\end{array}$} & \multirow{2}{*}{$\begin{array}{c}\begin{array}{c}\text { Coeficientes } \\
\text { padronizados }\end{array} \\
\text { Beta }\end{array}$} & \multirow[b]{2}{*}{$\mathrm{t}$} & \multirow[b]{2}{*}{ Sig. } & \multicolumn{2}{|c|}{$\begin{array}{c}\text { Estatísticas de } \\
\text { colinearidade }\end{array}$} \\
\hline & B & Erro Padrão & & & & Tolerância & VIF \\
\hline (Constante) & 3,298 & ,264 & & 12,484 & 000 & & \\
\hline Q1 &,- 004 &, 054 &,- 006 &,- 067 & ,946 & ,592 & 1,691 \\
\hline Q2 &, 054 & ,047 & ,101 & 1,146 & ,253 &, 567 & 1,764 \\
\hline Q3 & , 152 & ,082 & , 126 & 1,867 & 063 & ,976 & 1,025 \\
\hline Q4 & ,083 & 040 & , 159 & 2,064 & 040 & ,747 & 1,340 \\
\hline Q5 &,- 002 &, 043 &,- 003 &,- 046 & ,963 &, 815 & 1,227 \\
\hline Q6 & ,047 & ,035 & ,104 & 1,340 &, 182 & ,737 & 1,357 \\
\hline Q7 &,- 022 &, 044 &,- 045 &,- 515 & ,607 &, 574 & 1,742 \\
\hline Q8 &,- 059 & ,051 &,- 112 & $-1,155$ & ,249 & ,474 & 2,108 \\
\hline Q9 &, 025 & ,046 & ,047 &, 542 &, 588 &, 585 & 1,710 \\
\hline
\end{tabular}

Fonte: Dados da Pesquisa (2018) 
De acordo com Hair Jr. et al. (2009), uma variável só é significante estatisticamente se o p-value estiver abaixo de 0,05. Nesse caso, conforme a Tabela 9, análise de variância demonstrou que apenas a variável independente Q4 é significante. A variável Q3 apresentouse marginalmente significante. As variáveis das questões Q1 e Q5 não apresentaram índices significativos para o modelo. A Q1 refere-se a dimensão distância do poder e a questão Q5 refere-se a dimensão individualismo versus coletivismo.

$\mathrm{Na}$ dimensão individualismo versus coletivismo, os alunos apresentaram um perfil mais individualista, acreditando que os objetivos dos grupos são mais relevantes que os seus objetivos pessoais. Segundo Hofstede (1991), os estudantes em uma cultura individualista pretendem ser tratados com imparcialidade e as amizades se formam de acordo com os interesses e habilidades em comum.

Por meio do nível de significância observa-se que a Q3 - Nossa família deve evitar se envolver com pessoas de outra classe social - possui uma significância de 0,63. Este valor indica que os adolescentes se posicionam contra a diferenciação por classe social, reforçando as características de Baixa distância do Poder e que as desigualdades entre as pessoas devem ser minimizadas.

Observando a ANOVA, na Q4 - Eu devo esquecer (abrir mão) do que quero se isso prejudicar meu grupo de estudos (trabalhos de escola) - verifica-se a tendência do adolescente em não abrir mão dos interesses individuais em prol do interesse coletivo, reforçando o perfil individualista. Cabe ressaltar que no estudo desenvolvido por Gonçalves et al. (2016) a mesma questão também apresentou fator significante.

Realizado o teste de inflação de variância, verificou-se que todas estavam abaixo de 5, o que demonstra baixa correlação entre as variáveis independentes e todos os valores de tolerância próximas de 1. Estes índices mostram um resultado positivo, e que não há colinearidade entre as variáveis independentes. O modelo estatístico apresentou melhor resultado nos índices de tolerância e de fator de inflação da variância (VIF) na variável Q3 (Tolerância ,976 e o VIF 1,025). Novamente a variável Q3 - Nossa família deve evitar se envolver com pessoas de outra classe social - apresentou os melhores índices, corroborando ainda mais com os resultados.

A Tabela 10 apresenta a correlação entre a variável dependente com as independentes (R) e o seu grau de explicação $\left(\mathrm{R}^{2}\right)$.

Tabela $10-\mathrm{R}^{2}$

\begin{tabular}{lcccc}
\hline & \multicolumn{2}{c}{ R quadrado - Regressões } & & \\
\hline Modelo & R quadrado & R quadrado ajustado & $\begin{array}{c}\text { Erro padrão da } \\
\text { estimativa }\end{array}$ & Durbin-Watson \\
\hline Particular Florianópolis &, 057 &, 017 &, 8914 & 1,826 \\
\hline
\end{tabular}

Fonte: Dados da Pesquisa (2018)

Segundo Koufteros (1999), um nível adequado de $\mathrm{R}^{2}$ deve ser acima de 0,5. Diante disto, constata-se que o grau de determinação desta pesquisa é aceitável, entre a variável independente (Inovatividade) com as dependentes (Distância do Poder: Q1, Q2, Q3 e Coletivismo: Q4, Q5, Q6, Q7, Q8, Q9). 


\section{CONCLUSÃO}

O presente estudo buscou identificar como a cultura influencia na adoção de inovações tecnológicas, entre os jovens da rede privada de Florianópolis, por meio das dimensões de individualismo versus coletivismo e distância do poder. Para tal, foi realizada uma pesquisa com a aplicação de 250 questionários entre os alunos do $1^{\circ}$ ao $3^{\circ}$ ano do ensino médio de uma rede de escolas privadas na cidade de Florianópolis.

Inicialmente como perfil dos estudantes, constatamos que a faixa etária dos respondentes variou entre 16 e 25 anos. Aparece um dado importante na pesquisa que é a quantia de $58,38 \%$ dos estudantes terem mais que dezoito anos. Segundo a direção da escola este fato ocorre pois muitos buscam novamente o terceiro ano do segundo grau como um reforço pré-vestibular. Sobre o grau de instrução dos familiares, se somados pais e mães, $80,4 \%$ dos pais enquadram-se no perfil com ensino superior ou pós-graduação. Entre os alunos da rede privada, as mães dos estudantes possuem um maior grau de instrução do que os pais.

$\mathrm{Na}$ Dimensão de Distância do Poder que envolvem Q1, Q2 e Q3, 81\% dos respondentes discordam da alta distância do poder dentro de casa. Chama a atenção a resposta da Q3, onde 97,6\% dos estudantes discordam no quesito que a família deve evitar se envolver com pessoas de outra classe social. Este resultado demonstra que o estudante não concorda com a distância do poder e que as desigualdades entre as pessoas devem ser minimizadas. $\mathrm{Na}$ análise de variância este resultado é reforçado com o maior índice de significância, aparecendo na ANOVA com uma Sig de 0,63.

Na Dimensão Individualismo versus Coletivismo que envolve Q4 a Q9 constatou-se a tendência dos estudantes da rede privada de educação a uma cultura mais individualista. A tendência dos estudantes a esta cultura individualista fica mais clara na questão 5 onde quase $83,5 \%$ dos estudantes discordam que devem deixar de lado o que querem para seguir o que seu grupo de estudos quer. $\mathrm{Na}$ análise de variância, a Q4 apresentou o melhor resultado com um Sig de 0,40. Esta afirmativa e seu resultado permitem afirmar que o estudante possui um perfil individualista.

A terceira parte do questionário visa medir o nível de adoção tecnológica por jovens. Esta etapa do questionário apresentou uma média de 3,70 pontos na escala tipo Likert, ou seja, com maior propensão à adoção de inovações tecnológicas, inclusive os maiores resultados foram obtidos nas questões sobre exploração e experimentação de novas tecnologias.

Diante dos resultados pode-se supor que o jovem estudante da rede privada de Florianópolis tem um perfil menos tolerante a distância do poder, possui uma cultura individualista e é propenso a adoção de inovações tecnológicas, principalmente na experimentação de novidades.

Como limitação deste estudo está o corte transversal e aplicação do questionário em uma rede de escolas privadas. Como recomendação, elencamos a possibilidade de comparar os estudantes da Capital do Estado de Santa Catarina com estudantes de outras cidades e características diferentes para identificar o perfil destes jovens. 


\section{REFERÊNCIAS}

AFUAH, Allan. Redefining firm boundaries in the face of the internet: are firms really shrinking? Academy of Management Review, v. 28, n. 1, p. 34-53, 2003.

BECKER, Howard S.; GEER, Blanche. Latent culture: A note on the theory of latent social roles. Administrative Science Quarterly, v. 5, n. 2, p. 304-313, 1960.

BRESCHI, Stefano; MALERBA, Franco. Sectoral innovation systems: technological regimes, Schumpeterian dynamics, and spatial boundaries. Systems of innovation: Technologies, institutions and organizations, p. 130-156, 1997.

CASAGRANDE JR, Eloy Fassi. Inovação tecnológica e sustentabilidade: integrando as partes para proteger o todo. Palestra do Seminário de Tecnologia-PPGTE-CEFET-PR: Curitiba, 2003.

DAMANPOUR, Fariborz. Organizational innovation: A meta-analysis of effects of determinants and moderators. Academy of management journal, v. 34, n. 3, p. 555-590, 1991.

DE SOUZA PIRES, José Calixto; MACÊDO, Kátia Barbosa. Cultura organizacional em organizações públicas no Brasil. Revista de Administração Pública, v. 40, n. 1, p. 81-106, 2006.

DOSI, Giovanni. Sources, procedures, and microeconomic effects of innovation. Journal of economic literature, p. 1120-1171, 1988.

DOWNS JR, George W.; MOHR, Lawrence B. Conceptual issues in the study of innovation. Administrative science quarterly, p. 700-714, 1976.

GONÇALVES, Andressa et al. O impacto da cultura no uso de produtos com tecnologia agregada: estudo com adolescentes. REGEPE-Revista de Empreendedorismo e Gestão de Pequenas Empresas, v. 5, n. 1, p. 192-216, 2016.

GUNDLING, Ernest. How to communicate globally. Training \& Development, v. 53, n. 6, p. 28-32, 1999.

HAMEL, Gary. The why, what, and how of management innovation. Harvard business review, v. 84, n. 2, p. 72, 2006.

HAIR, JR. J. F. et al. Fundamentos de métodos de pesquisa em administração. São Paulo: Saraiva, 2005.

HOFSTEDE, Geert. Cultures and organizations. Intercultural cooperation and its importance for survival. Software of the mind. London: Mc Iraw-Hill, 1991.

HOFSTEDE, Geert. Dimensionalizing cultures: The Hofstede model in context. Online readings in psychology and culture, v. 2, n. 1, p. 8, 2011. 
ISMAIL, Wan Khairuzzaman Wan; ABDMAJID, R. Framework of the culture of innovation: a revisit. Jurnal Kemanusiaan, v. 5, n. 1, 2007.

JO HATCH, Mary; SCHULTZ, Majken. Relations between organizational culture, identity and image. European Journal of marketing, v. 31, n. 5/6, p. 356-365, 1997.

KOUFTEROS, Xenophon A. Testing a model of pull production: a paradigm for manufacturing research using structural equation modeling. Journal of Operations Management, v. 17, n. 4, p. 467-488, 1999.

NICOLACI-DA-COSTA, Ana Maria. Sociabilidade virtual: separando o joio do trigo. Psicologia \& Sociedade, v. 17, n. 2, p. 50-57, 2005.

MACHADO, Denise Del Prá Netto; CARVALHO, Carlos Eduardo. Traços culturais de pequenas empresas do setor madeireiro. Revista Ciências Administrativas ou Journal of Administrative Sciences, v. 12, n. 1, p. 16-24, 2006.

MALHOTRA, N. K. Pesquisa de marketing: uma orientação aplicada. 3.ed. Porto Alegre: Ed. Bookman, 2001

MATIAS-PEREIRA, J.; KRUGLIANSKAS, I. Gestão de Inovação: a lei de inovação tecnológica como ferramenta de apoio às políticas industrial e tecnológica do Brasil. RAE, v. 4, n. 2, Art. 18, jul./dez. 2005

OCDE. Manual de Oslo: diretrizes para coleta e interpretação de dados sobre inovação. $3^{\mathrm{a}}$. Ed. Brasília, Finep, 2005. Disponível em: <http://http://www.petrobras.com.br/minisite/ comunidade_cienciatecnologia/portugues/docs/Manual-de-Oslo.pdf $>$. Acesso em: 21 jun. 2018.

PARASURAMAN, Ananthanarayanan; GREWAL, Dhruv. The impact of technology on the quality-value-loyalty chain: a research agenda. Journal of the academy of marketing science, v. 28, n. 1, p. 168-174, 2000.

PETTIGREW, Andrew M. On studying organizational cultures. Administrative science quarterly, v. 24, n. 4, p. 570-581, 1979.

ROTHWELL, Roy; GARDINER, Paul. Invention, innovation, re-innovation and the role of the user: a case study of British hovercraft development. Technovation, v. 3, n. 3, p. 167-186, 1985.

SCHUMPETER, Joseph A.; BECKER, Markus C.; KNUDSEN, Thorbjorn. Entrepreneur. In: Austrian Economics and Entrepreneurial Studies. Emerald Group Publishing Limited, 2003, p. 235-265.

TIDD, Joe; BESSANT, John; PAVITT, Keith. Managing innovation integrating technological, market and organizational change. John Wiley and Sons Ltd, 2005.

VAN DE VEN, Andrew H.; ANGLE, Harold L. An introduction to the Minnesota innovation research program. Research on the management of innovation, p. 3-30, 1989. 
VARANDAS JUNIOR, Angelo et al. Análise da gestão da cadeia de valor da inovação em uma empresa do setor siderúrgico. Gestão \& Produção, v. 21, n. 1, p. 1-18, 2014.

VARGAS, Katiuscia Schiemer; SCHIAVINI, Janaina Mortari; DA ROSA SCHREINER, Janaína Schiemer. Inovação tecnológica como forma de desenvolvimento sustentável no setor de saneamento ambiental. Revista Espacios, v. 33, n. 11, 2012.

VASCONCELLOS, Marcos Augusto de. Introdução. In.: BARBIERI, José Carlos. Organizações inovadoras: estudos e casos brasileiros. Rio de Janeiro: Ed. FGV, 2003, p. 1330 .

ZALTMAN, Gerald; DUNCAN, Robert; HOLBECK, Jonny. Innovativeness and Organizations. NY: John Wiley and Sons, 1973.

Artigo recebido em: 06/09/2018

Artigo aprovado em: 13/09/2018 